\title{
PIECEWISE BOUNDED QUADRATIC SYSTEMS IN THE PLANE
}

\author{
JAUME LLIBRE, CLAYTON E. L. DA SILVA AND PAULO R. DA SILVA
}

\begin{abstract}
In this paper we study the sliding mode of piecewise bounded quadratic systems in the plane given by a non-smooth vector field $Z=(X, Y)$. Analyzing the singular, crossing and sliding sets, we get the conditions which ensure that any solution, including the sliding one, is bounded.
\end{abstract}

\section{INTRODUCTION}

Non-smooth dynamical systems appear in a large number of problems from mechanics (dry friction with stick and slip modes, impacts, oscillating systems with viscous damping, elasto plasticity), electrical engineering (electrical circuits and networks with switches, power electronics) theory of automatic and optimal control, games theory, walking machines, biological and physiological systems. For bidimensional problems some of these models are described by piecewise differential system (or non-smooth system) as follows

$$
\dot{x}= \begin{cases}X(x) & \text { if } \quad f(x) \geqslant 0, \\ Y(x) & \text { if } \quad f(x) \leqslant 0\end{cases}
$$

where $f: \Omega \subset \mathbb{R}^{2} \longrightarrow \mathbb{R}$ and $X, Y: \Omega \subset \mathbb{R}^{2} \longrightarrow \mathbb{R}^{2}$ are of class $C^{r}(r \geqslant 1)$ and 0 is a regular value of $f$. The dynamics of the system on the switching manifold $\Sigma$ given by the points $x$ such that $f(x)=0$ is also studied. We use the notation $Z(x)=(X(x), Y(x))$ or simply $Z=(X, Y)$ and assume that $Z$ is bi-valuated on $\Sigma$. The flow of $Z=(X, Y)$ is defined respectively by the vector fields $X$ and $Y$ as usual. The convention adopted by Filippov, in the pioneering work [4], for the transition of orbits (trajectories) between the two regions separated by $\Sigma$ and its permanence in $\Sigma$ is presented in section 2 .

In this work we consider that $X$ and $Y$ are bounded quadratic vector fields in the plane. More precisely we study $X$ and $Y$ as one of the normal forms of the bounded quadratic vector fields, studied in [6].

2010 Mathematics Subject Classification. 34C15, 34K12, 34H05.

Key words and phrases. non-smooth dynamical systems, quadratic systems, bounded quadratic systems, sliding vector fields, phase portrait.

* The first author is partially supported by a MINECO/ FEDER grant number MTM200803437, by a AGAUR grant number 2009 SGR 410, by ICREA Academia and by FP7-PEOPLE2012-IRSES 316338 and 318999. The second author is supported by CAPES, the third author is partially supported by CNPq, FAPESP and FP7-PEOPLE-2012-IRSES 318999. All authors are partially supported by the project PHB 2009-0025-PC.. 
A piecewise differential system is said bounded when all its trajectories are bounded for $t \geqslant 0$. A natural question is

$$
X, Y \text { bounded } \Longrightarrow Z=(X, Y) \text { bounded? }
$$

The following proposition says that in general the answer is negative.

Proposition 1. There exist bound quadratic vector fields $X$ an $Y$ such that $Z=(X, Y)$ is not bounded.

In this paper we consider the following:

Problem: Let $Z=(X, Y)$ be a non-smooth vector field with $X$ and $Y$ bounded quadratic. Is it possible to give sufficient conditions on the coefficients of the linear parts of $X$ and $Y$ which ensures that $Z$ is bounded?

The paper is organized as follows. In section 2 we present preliminaries results and state our main results. In section 3 we prove the main results. In section 4 we present an important application of the piecewise bounded quadratic system: the mass-spring system. This model is extremely important for the study of the natural phenomena. It is used as a good approximation for small amplitude oscillations. The oscillatory systems can be studied by second order differential equations, from the application of laws physical, such as Newton's laws, Hooke's law and hypothesis such as damping proportional to the speed etc. We analyzed the mass-springer system under the action of a force with different intensities and the switching manifold $\Sigma$ is interpreted as the interrupt of each one of the forces to start the other. For more details see section 4 .

\section{Preliminaries}

2.1. Bounded quadratic systems in the plane. Consider an autonomous quadratic differential system in the plane with an equilibrium point at $(0,0)$ given by

$$
\dot{x}=A x+\varphi(x),
$$

with $x=\left(x_{1}, x_{2}\right) \in \mathbb{R}^{2}, A=\left(a_{i j}\right)_{2 \times 2}$ a real constant matrix, $\varphi(x)=(P(x), Q(x))^{T}$, $P(x)=a x_{1}^{2}+b x_{1} x_{2}+c x_{2}^{2}$ and $Q(x)=d x_{1}^{2}+e x_{1} x_{2}+f x_{2}^{2}$ where $a, b, c, d, e, f$ are real constants.

According to Markus (see [6]) if $\varphi(x) \neq 0$ it is linearly equivalent to one of the following forms:

(2) $\left(0, x_{1} x_{2}\right)^{T} ; \quad\left(x_{2}^{2}, 0\right)^{T} ; \quad\left(x_{1} x_{2}+x_{2}^{2}, x_{2}^{2}\right)^{T} ; \quad\left(x_{2}^{2},-x_{1} x_{2}+c x_{2}^{2}\right)^{T}, \quad|c|<2$.

We say that a quadratic system is bounded if all its trajectories are bounded for $t \geqslant 0$. Dickson and Perko (see [3]) determined what of those systems (1) are bounded. More preciselly they proved the following.

Theorem 2. Consider $\dot{x}=A x+\varphi(x)$ an autonomous quadratic differential system in the plane with an equilibrium point at $(0,0)$, as above, and initial condition $x(0)=x_{0}$. 
(a) If $\varphi(x)=\left(x_{1} x_{2}+x_{2}^{2}, x_{2}^{2}\right)^{T}$, then the system has an unbounded trajectory (as $t \rightarrow \infty$ ) for some $x_{0} \in \mathbb{R}^{2}$.

(b) If $\varphi(x)=\left(0, x_{1} x_{2}\right)^{T}$, then the system has all of its trajectories bounded (for $t \geqslant 0$ ) if and only if $a_{12}=0, a_{11}<0$ and $a_{22} \leqslant 0$.

(c) If $\varphi(x)=\left(x_{2}^{2}, 0\right)^{T}$, then the system has all of its trajectories bounded (for $t \geqslant 0)$ if and only if $a_{21}=0, a_{11} \leqslant 0, a_{22} \leqslant 0$ and $a_{11}+a_{22}<0$.

(d) If $\varphi(x)=\left(x_{2}^{2},-x_{1} x_{2}+c x_{2}^{2}\right)^{T}$, then the system has all of its trajectories bounded (for $t \geqslant 0$ ) if and only if $|c|<2$ and satisfies one of the following sets of conditions: (i) $a_{11}<0$; (ii) $a_{11}=0$ and $a_{21}=0$; or (iii) $a_{11}=0, a_{21} \neq 0$, $a_{12}+a_{21}=0$ and $c a_{21}+a_{22} \leqslant 0$.

Theorem 2 classifies the quadratic systems in the plane that are bounded. If a quadratic system is linearly equivalent to a system with the quadratic part given by $\left(0, x_{1} x_{2}\right)^{T},\left(x_{2}^{2}, 0\right)^{T}$ or $\left(x_{2}^{2},-x_{1} x_{2}+c x_{2}^{2}\right)^{T}$, satisfying the respective conditions of the statements $(b),(c)$ or $(d)$, then this system is bounded.

The complete study of the phase portraits of the bounded quadratic systems having limit cycles can be found in [1] and their perturbation inside all quadratic systems in [5]. Moreover we refer [2] as a survey of the known results for this kind of systems.

First proof of Proposition 1. Consider $f\left(x_{1}, x_{2}\right)=x_{2}$ and $Z=(X, Y)$ with

$$
X\left(x_{1}, x_{2}\right)=\left(2+3 x_{2}+x_{2}^{2},-1-x_{2}\right) \quad \text { and } \quad Y\left(x_{1}, x_{2}\right)=\left(-1+x_{2}^{2}, 2-2 x_{2}\right) .
$$

Applying Theorem 2, statement (c), we conclude that $X$ and $Y$ are bounded. The manifold $\Sigma$, that is formed by the points $\left(x_{1}, 0\right)$, is a sliding region and the vector field defined in this region, called Filippov vector field, is $F_{Z}\left(x_{1}, 0\right)=(1,0)$. This vector field has no equilibrium point and the trajectory of $F_{Z}$ is unbounded, as shown in figure 1. Thus $Z=(X, Y)$ is unbounded. For details see section 2 .

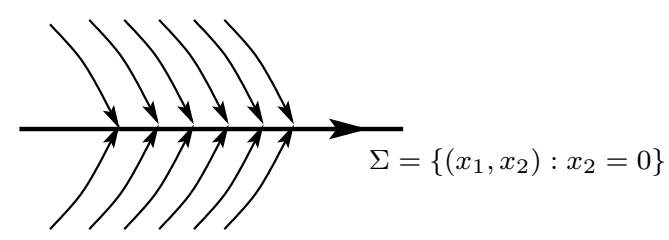

FIGURE 1. Unbounded non-smooth system in the sliding region.

2.2. Piecewise smooth dynamical systems in $\mathbb{R}^{2}$. Let $f: \Omega \subset \mathbb{R}^{2} \longrightarrow \mathbb{R}$ be a smooth function and $\Omega$ an open set. Consider $\Sigma=f^{-1}(0)=\{x \in \Omega: f(x)=$ $0\}, \Sigma^{+}=\{x \in \Omega: f(x) \geqslant 0\}$ and $\Sigma^{-}=\{x \in \Omega: f(x) \leqslant 0\}$. Assume that 0 is a regular value of $f$, i.e., $\nabla f(x) \neq(0,0)$, for any $x \in \Sigma$. As usual $\nabla f$ denotes the gradient vector $\nabla f=\left(\partial f / \partial x_{1}, \partial f / \partial x_{2}\right)$. 
We denote $\chi^{r}(\Omega)$ the set of the vector fields $X: \Omega \subset \mathbb{R}^{2} \longrightarrow \mathbb{R}^{2}$ of $C^{r}$ class with $r \geqslant 1$.

Consider $X, Y \in \chi^{r}(\Omega)$. A vector field like the following

$$
Z(x)=\left\{\begin{array}{lll}
X(x) & \text { if } & x \in \Sigma^{+}, \\
Y(x) & \text { if } & x \in \Sigma^{-},
\end{array}\right.
$$

is said to be a non-smooth vector field with switching manifold $\Sigma$, and it is denoted by $Z=(X, Y)$. The set of these vector fields is denoted for $\chi^{r}(\Omega, f)$.

Define $X f(x):=\langle X(x), \nabla f(x)\rangle$ and $Y f(x):=\langle Y(x), \nabla f(x)\rangle$ for any $x \in \Sigma$.

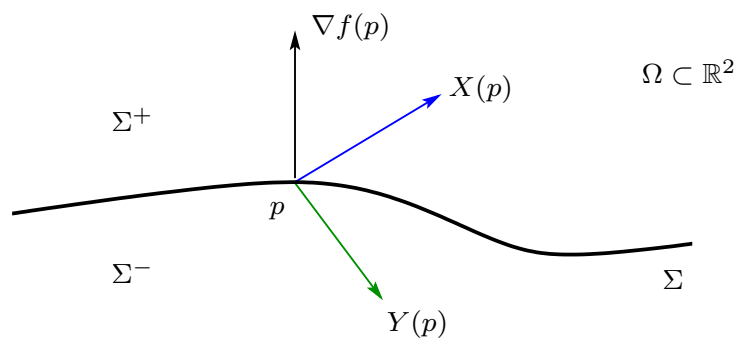

Figure 2. Non-smooth vector field $Z=(X, Y)$ in $p$ with $X f(p)>0$ and $Y f(p)<0$.

We classify the points on $\Sigma$ according the product $X f(x) \cdot Y f(x)$. By the Filippov convention, the points $x \in \Sigma$ such that $X f(x) \cdot Y f(x)>0$ determine a crossing region and the points $x \in \Sigma$ such that $X f(x) \cdot Y f(x)<0$ determine a sliding region. The points $x \in \Sigma$ such that $X f(x) \cdot Y f(x)=0$ are called tangent points of $Z$. Thus we have $\Sigma=\Sigma_{c} \cup \Sigma_{s} \cup \Sigma_{t}$, where $\Sigma_{c}=\{x \in \Sigma: X f(x) \cdot Y f(x)>$ $0\}, \Sigma_{s}=\{x \in \Sigma: X f(x) \cdot Y f(x)<0\}$ and $\Sigma_{t}=\{x \in \Sigma: X f(x) \cdot Y f(x)=0\}$.

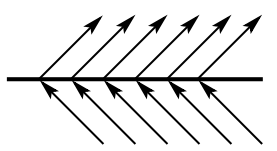

(a) $X f, Y f>0$

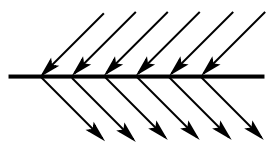

(b) $X f, Y f<0$

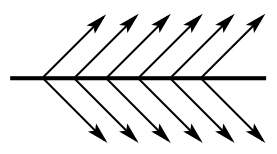

(c) $X f>0, Y f<0$

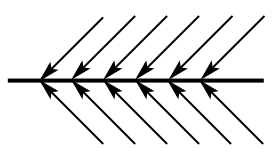

(d) $X f<0, Y f>0$

FiguRE 3. Examples of the crossing regions in (a) and (b), and sliding regions in (c) and (d).

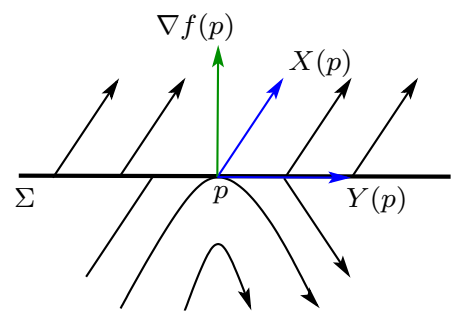

Figure 4. Tangent point $p$ with $Y f(p)=0$, separating the crossing and sliding regions.

In the sliding region, we define the Filippov vector field, which is given by

$$
F_{Z}(x)=\lambda X(x)+(1-\lambda) Y(x),
$$


for any $x \in \Sigma_{s}$ with $\lambda \in[0,1]$ satisfying $\langle\lambda X(x)+(1-\lambda) Y(x), \nabla f(x)\rangle=0$, as shown in figure 5 .

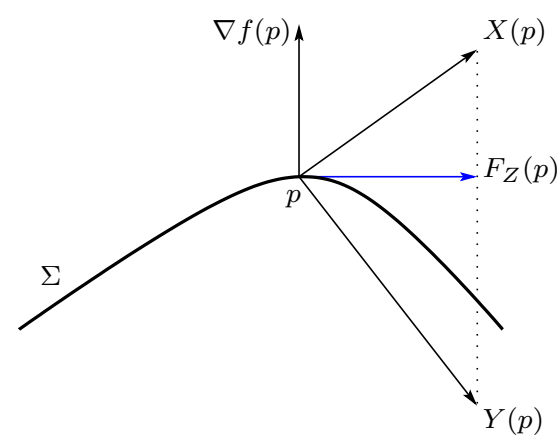

FiguRE 5. The Filippov vector field.

We say that a point $p \in \Sigma$ is a regular point of $Z$ if $p \in \Sigma_{c}$ or if $p \in \Sigma_{s}$ and $\operatorname{det}[X, Y](p) \neq 0$, i.e., $\{X(p), Y(p)\}$ is a linearly independent set.

An equilibrium point of $F_{Z}$ is a point $p \in \Sigma_{s}$ such that $\operatorname{det}[X, Y](p)=0$. This equilibrium point is called hyperbolic if $d(\operatorname{det}[X, Y])(p) \neq 0$. If $d(\operatorname{det}[X, Y])(p)>$ 0 then the hyperbolic equilibrium point $p$ is a saddle if $X f(p)<0$ and $Y f(p)>0$ or a repelling node if $X f(p)>0$ and $Y f(p)<0$. If $d(\operatorname{det}[X, Y])(p)<0$ then $p$ is a saddle if $X f(p)>0$ and $Y f(p)<0$ or a attracting node if $X f(p)<0$ and $Y f(p)>0$. The set of the equilibrium points of $F_{Z}$ is denoted by $\Sigma_{e}$.

We say that a point $p \in \Sigma$ is a singular point of $Z$ if $p$ is a tangent point of $Z$ or $p$ is an equilibrium point of $F_{Z}$.

The set of the regular points is denoted by $\Sigma^{r e g}$ and the set of the singular points is denoted for $\Sigma^{s i n}$. Thus $\Sigma^{r e g}=\Sigma_{c} \cup\left(\Sigma_{s} \backslash \Sigma_{e}\right)$ and $\Sigma^{s i n}=\Sigma_{t} \cup \Sigma_{e}$.

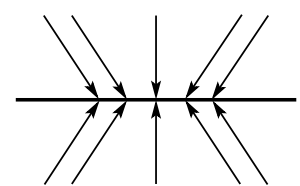

(a) Attracting node

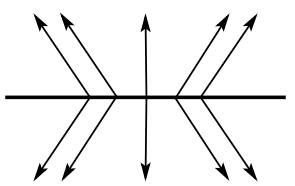

(b) Repelling node

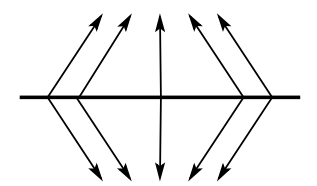

(c) Saddle

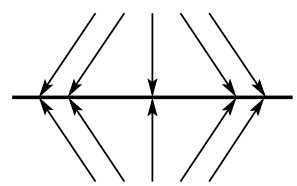

(d) Saddle

FigURE 6. Hyperbolic equilibrium points.

2.3. Piecewise bounded quadratic systems in the plane. Let $Q^{r}(\Omega, f) \subset$ $\chi^{r}(\Omega, f)$ be the set of piecewise quadratic systems in the plane. Consider $x=$ $\left(x_{1}, x_{2}\right) \in \mathbb{R}^{2}, p=\left(p_{1}, p_{2}\right) \in \mathbb{R}^{2}, q=\left(q_{1}, q_{2}\right) \in \mathbb{R}^{2}$ and $A=\left(a_{i j}\right)_{2 \times 2}, B=$ $\left(b_{i j}\right)_{2 \times 2}, \varphi$ and $\psi$ as in (1). In this subsection and in the following we consider $\Sigma=f^{-1}(0)$ with $f(x)=x_{2}$ and $Z=(X, Y)$ given by

$$
Z(x)=\left\{\begin{array}{lll}
X(x)=A(x-p)+\varphi(x-p) & \text { if } \quad x_{2} \geqslant 0 \\
Y(x)=B(x-q)+\psi(x-q) & \text { if } \quad x_{2} \leqslant 0 .
\end{array}\right.
$$

Observe that the equilibrium point of $X$ and $Y$ are now the points $p$ and $q$ respectively and $\Sigma=\left\{\left(x_{1}, x_{2}\right) \in \mathbb{R}^{2}: x_{2}=0\right\}$. 


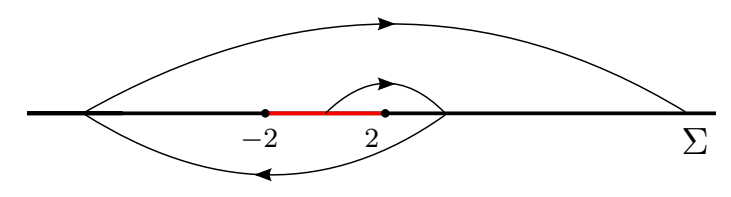

Figure 7. An unbounded trajectory of $Z=(X, Y)$ passing by crossing points, with $X$ and $Y$ bounded.

Similarly as defined in section 2.1, we say that a piecewise quadratic system in the plane is bounded if all its trajectories are bounded for $t \geqslant 0$.

In the introduction we mentioned that if $X$ and $Y$ are bounded quadratic vector fields, this does not imply that $Z=(X, Y)$ is bounded and the example that was presented is unbounded in the sliding region $\Sigma_{s}$.

The vector field $Z=(X, Y)$ with $X$ and $Y$ bounded, can have an unbounded trajectory $x(t)=\left(x_{1}(t), x_{2}(t)\right)$ passing by crossing points as shown the example below.

Second proof of Proposition 1. Consider the vector field $Z \in Q^{r}(\Omega, f)$ given by

$$
Z\left(x_{1}, x_{2}\right)=\left\{\begin{array}{l}
\left(\begin{array}{ll}
0 & 0 \\
0 & 1
\end{array}\right)\left(\begin{array}{l}
x_{1}-1 \\
x_{2}+1
\end{array}\right)+\left(\begin{array}{c}
\left(x_{2}+1\right)^{2} \\
-\left(x_{1}-1\right)\left(x_{2}+1\right)
\end{array}\right) \quad \text { if } x_{2} \geqslant 0, \\
\left(\begin{array}{ll}
0 & 0 \\
0 & 1
\end{array}\right)\left(\begin{array}{l}
x_{1}+1 \\
x_{2}-1
\end{array}\right)-\left(\begin{array}{c}
\left(x_{2}-1\right)^{2} \\
-\left(x_{1}+1\right)\left(x_{2}-1\right)
\end{array}\right) \quad \text { if } \quad x_{2} \leqslant 0 .
\end{array}\right.
$$

The lines $x_{2}=-1$ and $x_{2}=1$ are lines of equilibrium points of $X$ and $Y$ respectively. We have $\Sigma_{t}=\{-2,2\}, \Sigma_{s}=\left\{x_{1} \in \mathbb{R}:-2<x_{1}<2\right\}$ and $\Sigma_{c}=\left\{x_{1} \in \mathbb{R}: x_{1}<-2\right.$ or $\left.x_{1}>2\right\}$. The vector field $Z$ is unbounded. See figure 7 .

Our main result is the following.

Theorem 3. Let $Z=(X, Y) \in Q^{r}(\Omega, f)$ be a non-smooth quadratic system with $X, Y$ bounded and $f\left(x_{1}, x_{2}\right)=x_{2}$.

(a) If either $\Sigma=\Sigma_{s}$, or $\sharp \Sigma_{t}=1$ and $\Sigma_{s}$ is unbounded, or $\sharp \Sigma_{t} \geq 2$ and $\Sigma_{C}$ is bounded, then $Z$ is bounded if and only if the sliding vector field $F_{Z}\left(x_{1}\right) \leq 0$ near $x_{1}=+\infty$ and $F_{Z}\left(x_{1}\right) \geq 0$ near $x_{1}=-\infty$.

(b) If $\Sigma=\Sigma_{C}$ then $Z$ is bounded.

Now consider $Z=(X, Y)$ where $X$ and $Y$ are given by $A x+\varphi(x)$ as in the Theorem 2 statements $(b),(c)$ or $(d)$. Let

$$
Q_{i}^{p}=\left\{F_{i} \in \chi^{r}(\Omega): F_{i}(x)=A^{(i)}(x-p)+\varphi^{(i)}(x-p)\right\} \text { for } i=1, \ldots, 5
$$

be the set of quadratic vector fields satisfying the following conditions

$A^{(1)}=\left(a_{i j}\right)_{2 \times 2}$ with $a_{11}<0, a_{12}=0, a_{22} \leqslant 0$;

$A^{(2)}=\left(a_{i j}\right)_{2 \times 2}$ with $a_{11} \leqslant 0, a_{21}=0, a_{22} \leqslant 0, a_{11}+a_{22}<0$;

$A^{(3)}=\left(a_{i j}\right)_{2 \times 2}$ with $a_{11}<0$;

$A^{(4)}=\left(a_{i j}\right)_{2 \times 2}$ with $a_{11}=a_{21}=0$;

$A^{(5)}=\left(a_{i j}\right)_{2 \times 2}$ with $a_{11}=0, a_{21} \neq 0, a_{12}+a_{21}=0, c a_{21}+a_{22} \leqslant 0$; 
$\varphi^{(1)}(y)=\left(0, y_{1} y_{2}\right)^{T}$

$\varphi^{(2)}(y)=\left(y_{2}^{2}, 0\right)^{T}$

$\varphi^{(3)}(y)=\varphi^{(4)}(y)=\varphi^{(5)}(y)=\left(y_{2}^{2},-y_{1} y_{2}+c y_{2}^{2}\right)^{T}$.

The constant $c$ in the expressions of $\varphi^{(3)}, \varphi^{(4)}, \varphi^{(5)}$ is such that $|c|<2$ according with Theorem 2 and $y=x-p$ with $y=\left(y_{1}, y_{2}\right)$.

Define $Q^{p}=\bigcup_{i=1}^{5} Q_{i}^{p}$. For $p, q \in \mathbb{R}^{2}$ denote $Q^{p q}=Q^{p} \times Q^{q}$. Thus $Q^{p q}=\bigcup_{i, j=1}^{5} B_{i j}$ with $B_{i j} \cap B_{k l}=\emptyset$ if $i \neq k$ or $j \neq l$ where $B_{i j}=Q_{i}^{p} \times Q_{j}^{q}$.

In what follows when we refer the coefficients of the matrix of the vector field $X$ we use $a_{i j}$ and for the vector field $Y$ we use $b_{i j}$.

Theorem 4. Let $Q^{p q}=Q^{p} \times Q^{q}$ be a subset of $Q^{r}(\Omega, f)$ as described above and $\Sigma=f^{-1}(0)$ where $f\left(x_{1}, x_{2}\right)=x_{2}$. There exists $B_{i j}^{L} \subset B_{i j}, B_{i j}^{L} \neq \emptyset$, such that any $Z=(X, Y) \in B_{i j}^{L}$ is bounded. Moreover the boundness is characterized by the sign of rational functions depending on the parameters $\left(a_{11}, a_{12}, a_{21}, a_{22}, b_{11}, b_{12}\right.$, $\left.b_{21}, b_{22}\right)$.

Theorem 4 characterizes the non-smooth vector fields $Z=(X, Y) \in Q^{p q}$ which are bounded. In section 3 we prove Theorems 3 and 4 . To prove Theorem 4 , first of all we compute $X f \cdot Y f$ on $\Sigma$. We prove (see Lemma 5 ) that either $X f \cdot Y f \equiv 0$ or $\Sigma$ has 0,1 or 2 tangent points. If $X f \cdot Y f \equiv 0$ then $Z$ is bounded. Besides, if $\Sigma$ has 0,1 or 2 tangent points then Lemmas 7, 8 and 11, respectively, give the conditions for the boundness.

\section{Proof of Theorems 3 And 4}

In this section we proof our main results.

Proof of Theorem 3. (a). Suppose that $\Sigma=\Sigma_{s}$. Thus the Filippov vector field $F_{Z}$ is an one dimensional vector field. Therefore $Z$ is bounded if and only if $F_{Z}\left(x_{1}\right) \leq 0$ near $x_{1}=+\infty$ and $F_{Z}\left(x_{1}\right) \geq 0$ near $x_{1}=-\infty$. If $\# \Sigma_{t}=1$ and $\Sigma_{s}$ is unbounded again the only possibility of $Z$ be unbounded, occurs in sliding region $\Sigma_{s}$ because if $Z$ has an unbounded trajectory passing by the crossing region $\Sigma_{c}$ then there exists at least two distinct points $(z, 0)$ and $(w, 0)$ of $\Sigma_{c}$ with $X f(z, 0), Y f(z, 0)>0$ and $X f(w, 0), Y f(w, 0)<0$. Thus exist $y^{t} \neq x^{t}$ such that $X f\left(y^{t}\right) \cdot Y f\left(y^{t}\right)=0$. The last case, $\Sigma=\Sigma_{s} \cup \Sigma_{c} \cup \Sigma_{t}$ with $\Sigma_{c}$ bounded, just make the study of the signal of $X f \cdot Y f$. The proof is analogous previous arguments $(b)$. If $\Sigma=\Sigma_{c}$ then the only possibility of a trajectory of $Z$ be unbounded occurs if it contains at least two distinct points of $\Sigma_{c}$. In this case there exists $z$ such that $X f(z, 0)=0$ or $Y f(z, 0)=0$ and thus $(z, 0) \in \Sigma_{t}$. Therefore $Z$ is bounded.

Proof of Theorem 4. In the following theorems we denote by $\Sigma_{t}^{0}$ the set of vector fields $Z \in Q^{p q}$ without tangent points, by $\Sigma_{t}^{1}$ with exactly one tangent point and by $\Sigma_{t}^{2}$ with two tangent points. We use the notation $[X f \cdot Y f]\left(x_{1}\right)$ to represent $X f\left(x_{1}, 0\right) \cdot Y f\left(x_{1}, 0\right)$ with $\left(x_{1}, 0\right) \in \Sigma$. 
Lemma 5. Consider $Z=(X, Y) \in Q^{p q}$. If $[X f \cdot Y f]\left(x_{1}\right) \neq 0$ for some $x_{1} \in \mathbb{R}$ then $\# \Sigma_{t}=0,1$ or 2 . Moreover, if $X f \cdot Y f \equiv 0$ then $Z$ is bounded.

Proof. Consider $Z=(X, Y) \in Q^{r}(\Omega, f)$ and suppose that $[X f \cdot Y f]\left(x_{1}\right) \neq 0$ for some $x_{1} \in \mathbb{R}$. Then $Z$ have at most 4 tangent points. In fact, the tangent points are solutions of equation $X f(x) \cdot Y f(x)=0$ with $x=\left(x_{1}, 0\right)$. Write

$$
Z(x)=\left\{\begin{array}{l}
\left(\begin{array}{ll}
a_{11} & a_{12} \\
a_{21} & a_{22}
\end{array}\right)\left(\begin{array}{l}
y_{1} \\
y_{2}
\end{array}\right)+\left(\begin{array}{l}
a_{1} y_{1}^{2}+a_{2} y_{1} y_{2}+a_{3} y_{2}^{2} \\
a_{4} y_{1}^{2}+a_{5} y_{1} y_{2}+a_{6} y_{2}^{2}
\end{array}\right) \quad \text { if } \quad x_{2} \geqslant 0, \\
\left(\begin{array}{ll}
b_{11} & b_{12} \\
b_{21} & b_{22}
\end{array}\right)\left(\begin{array}{l}
z_{1} \\
z_{2}
\end{array}\right)+\left(\begin{array}{l}
b_{1} z_{1}^{2}+b_{2} z_{1} z_{2}+b_{3} z_{2}^{2} \\
b_{4} z_{1}^{2}+b_{5} z_{1} z_{2}+b_{6} z_{2}^{2}
\end{array}\right) \quad \text { if } \quad x_{2} \leqslant 0
\end{array}\right.
$$

with $x=\left(x_{1}, x_{2}\right), y_{1}=x_{1}-p_{1}, y_{2}=x_{2}-p_{2}, z_{1}=x_{1}-q_{1}, z_{2}=x_{2}-q_{2}$ where $p=\left(p_{1}, p_{2}\right)$ and $q=\left(q_{1}, q_{2}\right)$ are equilibrium points of $X$ and $Y$ respectively. Thus

$$
\begin{gathered}
X f\left(x_{1}, 0\right)=\left\langle X\left(x_{1}, 0\right), \nabla f\left(x_{1}, 0\right)\right\rangle= \\
=a_{4} x_{1}^{2}+\left(a_{21}-2 a_{4} p_{1}-a_{5} p_{2}\right) x_{1}+\left(a_{4} p_{1}^{2}-a_{21} p_{1}-a_{22} p_{2}+a_{5} p_{1} p_{2}+a_{6} p_{2}^{2}\right),
\end{gathered}
$$

and

$$
\begin{gathered}
Y f\left(x_{1}, 0\right)=\left\langle Y\left(x_{1}, 0\right), \nabla f\left(x_{1}, 0\right)\right\rangle= \\
=b_{4} x_{1}^{2}+\left(b_{21}-2 b_{4} q_{1}-b_{5} q_{2}\right) x_{1}+\left(b_{4} q_{1}^{2}-b_{21} q_{1}-b_{22} q_{2}+b_{5} q_{1} q_{2}+b_{6} q_{2}^{2}\right) .
\end{gathered}
$$

Then

$$
\begin{gathered}
X f\left(x_{1}, 0\right) \cdot Y f\left(x_{1}, 0\right)=a_{4} b_{4} x_{1}^{4}+\left[a_{4}\left(b_{21}-2 b_{4} q_{1}-b_{5} q_{2}\right)+b_{4}\left(a_{21}-2 a_{4} p_{1}-a_{5} p_{2}\right)\right] x_{1}^{3}+ \\
+\left[a_{4}\left(b_{4} q_{1}^{2}-b_{21} q_{1}-b_{22} q_{2}+b_{5} q_{1} q_{2}+b_{6} q_{2}^{2}\right)+b_{4}\left(a_{4} p_{1}^{2}-a_{21} p_{1}-a_{22} p_{2}+a_{5} p_{1} p_{2}+a_{6} p_{2}^{2}\right)+\right. \\
\left.+\left(a_{21}-2 a_{4} p_{1}-a_{5} p_{2}\right)\left(b_{21}-2 b_{4} q_{1}-b_{5} q_{2}\right)\right] x_{1}^{2}+\ldots
\end{gathered}
$$

Note that $X f\left(x_{1}, 0\right) \cdot Y f\left(x_{1}, 0\right)$ is a polynomial of degree less than or equal to 4 depending on the coefficients. Thus it has at most 4 real zeros.

If $Z=(X, Y) \in Q^{p q}$ and $[X f \cdot Y f]\left(x_{1}\right) \neq 0$ for some $x_{1} \in \mathbb{R}$, then the nonsmooth vector field $Z$ has at most 2 tangent points. In fact it follows immediately since $a_{1}=a_{2}=a_{4}=b_{1}=b_{2}=b_{4}=0$ in each one of the cases for $Q_{i}^{p}$.

Now we prove the boundness of $Z$ when $X f \cdot Y f \equiv 0$ on $\Sigma$. In this case we conclude that $\Sigma=\Sigma_{t}$. If a trajectory of $(X, Y)$ is unbounded then either the trajectory passes by crossing region and returns or the trajectory escapes by sliding region. Since $\Sigma_{c}=\Sigma_{s}=\emptyset$, it can not happen.

Example 6. The number 4 in the proof of Lemma 5 is realizable. Choose the following: $a_{1}=a_{2}=a_{4}=a_{5}=b_{4}=a_{11}=a_{21}=b_{22}=p_{2}=q_{1}=1$, $a_{3}=a_{6}=-2, b_{1}=b_{12}=b_{21}=2, b_{2}=b_{5}=p_{1}=0, b_{3}=-21, b_{6}=-4$, $a_{12}=3, a_{22}=q_{2}=-1$ and $b_{11}=4$. Thus we obtain $\Sigma_{t}=\{ \pm 1, \pm \sqrt{3}\}$.

Lemma 7. Consider $Z \in Q^{p q} \cap \Sigma_{t}^{0}$. Then $\Sigma=\Sigma_{s}$ or $\Sigma=\Sigma_{c}$.

(a) If $\Sigma=\Sigma_{s}$ then $Z \in B_{i j}$ is bounded if and only if $R_{i j}^{p q}=A_{n} / B_{m}<0$ where $A_{n}$ is the coefficient of term of the greater degree of $\operatorname{det}[X, Y]\left(x_{1}, 0\right)$ with $n=\partial(\operatorname{det}[X, Y]), B_{m}$ is the coefficient of term of the greater degree of $Y_{2}\left(x_{1}, 0\right)-X_{2}\left(x_{1}, 0\right)$ with $m=\partial\left(Y_{2}-X_{2}\right)$ and $n \geqslant m$. If $\Sigma_{s}$ is composed only by equilibrium points of $F_{Z}$ then $Z$ is trivially bounded. 
(b) If $\Sigma=\Sigma_{c}$ then $Z$ is bounded.

Proof. By hypothesis $\# \Sigma_{t}=0\left(\Sigma_{t}=\emptyset\right)$. This imply that $\Sigma_{s}=\emptyset$ or $\Sigma_{c}=\emptyset$ otherwise (if $\Sigma_{s} \neq \emptyset$ and $\Sigma_{c} \neq \emptyset$ ) we would have at least one tangent point, since $X f \cdot Y f$ is a continuous function, $[X f \cdot Y f](z)>0$ and $[X f \cdot Y f](w)<0$ for $(z, 0) \in \Sigma$ and $(w, 0) \in \Sigma$. Therefore $\Sigma=\Sigma_{s}$ or $\Sigma=\Sigma_{c}$.

(a) We prove only the case $i=j=1$. The other cases are similar. We have $Z=(X, Y) \in B_{11}=Q_{1}^{p} \times Q_{1}^{q}$ where

$$
\begin{aligned}
& Q_{1}^{p}=\left\{F_{1} \in \chi^{r}(\Omega): F_{1}(x)=A^{(1)}(x-p)+\varphi^{(1)}(x-p)\right\}, \\
& Q_{1}^{q}=\left\{F_{1} \in \chi^{r}(\Omega): F_{1}(x)=B^{(1)}(x-q)+\varphi^{(1)}(x-q)\right\},
\end{aligned}
$$

and $A^{(1)}=\left(a_{i j}\right)_{2 \times 2}$ with $a_{11}<0, a_{12}=0, a_{22} \leqslant 0, B^{(1)}=\left(b_{i j}\right)_{2 \times 2}$ with $b_{11}<$ $0, b_{12}=0, b_{22} \leqslant 0, \varphi^{(1)}(y)=\left(0, y_{1} y_{2}\right)^{T}$ with $y=\left(y_{1}, y_{2}\right)$.

If $\Sigma=\Sigma_{s}$, the expression of the Filippov vector field $F_{Z}$ (seen as one dimensional) is

$$
\begin{gathered}
F_{Z}\left(x_{1}\right)= \\
\frac{\left(x_{1}-p_{1}\right)\left[\left(a_{11}\left(b_{21}-q_{2}\right)-b_{11}\left(a_{21}-p_{2}\right)\right)\left(x_{1}-q_{1}\right)-a_{11} b_{22} q_{2}\right]+b_{11} a_{22} p_{2}\left(x_{1}-q_{1}\right)}{\left[\left(b_{21}-q_{2}\right)-\left(a_{21}-p_{2}\right)\right] x_{1}-q_{1}\left(b_{21}-q_{2}\right)+p_{1}\left(a_{21}-p_{2}\right)-b_{22} q_{2}+a_{22} p_{2}} .
\end{gathered}
$$

Suppose that $b_{21} \neq q_{2}$ and $a_{21} \neq p_{2}$. Thus $A_{2}=a_{11}\left(b_{21}-q_{2}\right)-b_{11}\left(a_{21}-p_{2}\right)$ and $B_{1}=\left(b_{21}-q_{2}\right)-\left(a_{21}-p_{2}\right)$. We have

$$
\lim _{x_{1} \rightarrow \infty} F_{Z}\left(x_{1}\right)=\lim _{x_{1} \rightarrow \infty} \frac{a_{11}\left(b_{21}-q_{2}\right)-b_{11}\left(a_{21}-p_{2}\right)}{\left(b_{21}-q_{2}\right)-\left(a_{21}-p_{2}\right)} x_{1}
$$

where the symbol $\infty$ represents $\pm \infty$. Therefore $Z$ is bounded if and only if

$$
R_{11}^{p q}=\frac{a_{11}\left(b_{21}-q_{2}\right)-b_{11}\left(a_{21}-p_{2}\right)}{\left(b_{21}-q_{2}\right)-\left(a_{21}-p_{2}\right)}<0 .
$$

(b) If $\Sigma=\Sigma_{c}$ then $\Sigma_{t}=\Sigma_{s}=\emptyset$. The only possibility of a trajectory of $Z$ be unbounded occur if it contains at least two distinct points of $\Sigma_{c}$. If it occurs then there exists $z$ such that $X f(z, 0)=0$ or $Y f(z, 0)=0$ and thus $(z, 0) \in \Sigma_{t}$. Therefore $Z$ is bounded.

Lemma 8. Consider $Z \in Q^{p q} \cap \Sigma_{t}^{1}$. Then $\Sigma=\Sigma_{s} \cup \Sigma_{t} \cup \Sigma_{c}$ with $\Sigma_{s}$ and $\Sigma_{c}$ unbounded, $\Sigma=\Sigma_{s} \cup \Sigma_{t}$ or $\Sigma=\Sigma_{c} \cup \Sigma_{t}$. If $\Sigma=\Sigma_{s} \cup \Sigma_{t} \cup \Sigma_{c}$ with $\Sigma_{s}$ and $\Sigma_{c}$ unbounded or $\Sigma=\Sigma_{s} \cup \Sigma_{t}$ then $Z \in B_{i j}$ is bounded if and only if $R_{i j}^{p q}=A_{n} / B_{m}<0$ where $A_{n}$ is the coefficient of term of the greater degree of $\operatorname{det}[X, Y]\left(x_{1}, 0\right)$ with $n=\partial(\operatorname{det}[X, Y]), B_{m}$ is the coefficient of term of the greater degree of $Y_{2}\left(x_{1}, 0\right)-X_{2}\left(x_{1}, 0\right)$ with $m=\partial\left(Y_{2}-X_{2}\right)$ and $n \geqslant m$. If $\Sigma_{s}$ is composed only by equilibrium points of $F_{Z}$ then $Z$ is trivially bounded.

Proof. Since $\# \Sigma_{t}=1\left(\Sigma_{t}=\left\{x^{t}\right\}\right)$ then $[X f \cdot Y f]\left(x_{1}\right)$ is a polynomial of degree 1 or 2 . In the first case, we have $\Sigma=\Sigma_{s} \cup \Sigma_{t} \cup \Sigma_{c}$ with $\Sigma_{s}$ and $\Sigma_{c}$ unbounded. In the other case, $\Sigma=\Sigma_{s} \cup \Sigma_{t}$ or $\Sigma=\Sigma_{c} \cup \Sigma_{t}$. The only possibility of $Z$ be unbounded, occurs in sliding region $\Sigma_{s}$ because if $Z$ has an unbounded trajectory passing by the crossing region $\Sigma_{c}$ then there exists at least two distinct points $(z, 0)$ and $(w, 0)$ of $\Sigma_{c}$ with $X f(z, 0), Y f(z, 0)>0$ and $X f(w, 0), Y f(w, 0)<0$. 
Thus exist $y^{t} \neq x^{t}$ such that $X f\left(y^{t}\right) \cdot Y f\left(y^{t}\right)=0$. The remainder of proof is analogous to proof of the statement (a) of the Lemma 7.

Remark 9. If $\Sigma=\Sigma_{c} \cup \Sigma_{t}$ we can not ensure that the system is bounded. See the following example.

Example 10. Consider the vector field $Z$ given by

$$
Z\left(x_{1}, x_{2}\right)=\left\{\begin{array}{c}
E \cdot\left(\begin{array}{l}
x_{1}-1 \\
x_{2}+1
\end{array}\right)+\left(\begin{array}{c}
\left(x_{2}+1\right)^{2} \\
-\left(x_{1}-1\right)\left(x_{2}+1\right)+\left(x_{2}+1\right)^{2}
\end{array}\right) \quad \text { if } \quad x_{2} \geqslant 0, \\
F \cdot\left(\begin{array}{c}
x_{1}-3 \\
x_{2}-1
\end{array}\right)-\left(\begin{array}{c}
\left(x_{2}-1\right)^{2} \\
-\left(x_{1}-3\right)\left(x_{2}-1\right)+\left(x_{2}-1\right)^{2}
\end{array}\right) \quad \text { if } \quad x_{2} \leqslant 0,
\end{array}\right.
$$

with

$$
E=-F=\left(\begin{array}{ll}
0 & 0 \\
0 & 1
\end{array}\right) .
$$

The lines $x_{2}=-1$ and $x_{2}=1$ are lines of equilibrium points of $X$ and $Y$ respectively. We have $\Sigma_{t}=\{3\}, \Sigma_{s}=\emptyset$ and $\Sigma_{c}=\left\{x_{1} \in \mathbb{R}: x_{1} \neq 3\right\}$.

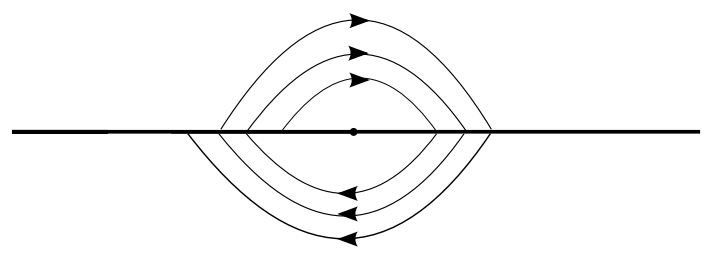

FiguRE 8. Unbounded trajectory when $\Sigma=\Sigma_{c} \cup \Sigma_{t}$.

Lemma 11. Consider $Z \in Q^{p q} \cap \Sigma_{t}^{2}$. Then $\Sigma=\Sigma_{s} \cup \Sigma_{c} \cup \Sigma_{t}$ with $\Sigma_{c}$ bounded or $\Sigma=\Sigma_{s} \cup \Sigma_{c} \cup \Sigma_{t}$ with $\Sigma_{s}$ bounded. If $\Sigma=\Sigma_{s} \cup \Sigma_{c} \cup \Sigma_{t}$ with $\Sigma_{c}$ bounded then $Z \in B_{i j}$ is bounded if and only if $R_{i j}^{p q}=A_{n} / B_{m}<0$ where $A_{n}$ is the coefficient of term of the greater degree of $\operatorname{det}[X, Y]\left(x_{1}, 0\right)$ with $n=\partial(\operatorname{det}[X, Y])$, $B_{m}$ is the coefficient of term of the greater degree of $Y_{2}\left(x_{1}, 0\right)-X_{2}\left(x_{1}, 0\right)$ with $m=\partial\left(Y_{2}-X_{2}\right)$ and $n \geqslant m$. If $\Sigma_{s}$ is composed only by equilibrium points of $F_{Z}$ then $Z$ is trivially bounded.

Proof. To verify that $\Sigma=\Sigma_{s} \cup \Sigma_{c} \cup \Sigma_{t}$ with $\Sigma_{c}$ bounded or $\Sigma=\Sigma_{s} \cup \Sigma_{c} \cup \Sigma_{t}$ with $\Sigma_{s}$ bounded, just make the study of the signal of $X f \cdot Y f$. The proof is analogous arguments to the proof of statement (a) of the Lemma 7.

Remark 12. If $\Sigma=\Sigma_{s} \cup \Sigma_{c} \cup \Sigma_{t}$ we cannot ensure that the system is bounded. See for instance, the example in the second proof of Proposition 1.

To define the set $B_{i j}^{L}$, stated in Theorem 4, we consider the set of quadratic systems $(X, Y) \in B_{i j}$ that satisfies the conditions given in the Lemmas $5,7,8$ and 11. It concludes the proof of Theorem 4 . 


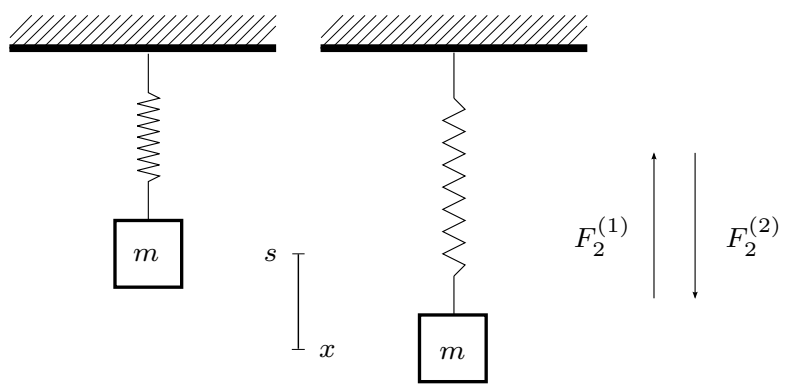

Figure 9. The mass-spring system under the action of two forces $F_{2}^{(1)}$ and $F_{2}^{(2)}$ given by quadratic term $y^{2}$ in the system (6).

\section{Application: the Mass-SPRing System}

Consider the vertical mass-spring system, as shown bellow. The object of mass $m$ when displaced from its equilibrium position $s$, experiences a restoring force $F_{1}$ proportional to the displacement $x$. This force is described as $F_{1}=-k(s+x)$ where $k$ is a positive constant, known as the spring constant. Suppose that a frictional force (damping), for example the air resistance, is also present. This force $F_{2}$ is proportional to the velocity and is described as $F_{2}=-\beta \dot{x}$, where $\beta$ is a positive constant. Consider the action of gravity $F_{3}=m g$, where $g \cong 9,8 \mathrm{~m} / \mathrm{s}^{2}$. The resultant force is $F=\sum F_{i}$ and using the Newton's second law and observing that $-k s=m g$ the we write

$$
m \ddot{x}=-k x-\beta \dot{x} .
$$

We want that the planar system to be quadratic and bounded and for this is sufficient choose $\dot{x}=y^{2}-c x$ with a convenient constant $c$. Thus we write the planar system

$$
\dot{x}=-c x+y^{2}, \quad \dot{y}=\frac{m c-\beta}{2 m} y-\frac{m c^{2}-\beta c+k}{2 m}(x / y) .
$$

Imposing the condition $m c^{2}-\beta c+k=0$, we find the values of $c$ such that the system (6) is quadratic. These values are

$$
c_{1}=\frac{\beta+\sqrt{\beta^{2}-4 m k}}{2 m} \quad \text { and } \quad c_{2}=\frac{\beta-\sqrt{\beta^{2}-4 m k}}{2 m} .
$$

We admit $\beta^{2}-4 m k>0$ and observe that $c_{1}, c_{2}>0$.

Now, consider $X(x, y)$ and $Y(x, y)$ the vector fields given by (6) with the respective constants $c_{1}$ and $c_{2}$. Observe that $\frac{m c_{1}-\beta}{2 m}$ and $\frac{m c_{2}-\beta}{2 m}$ are negative constants. Therefore, by Theorem 2, the quadratic systems given by $X$ and $Y$ are bounded.

Let $Z=(X, Y)$ be a non-smooth quadratic system with $f(x, y)=y$. Let $p=\left(p_{1}, p_{2}\right)$ be the equilibrium point of $X$ and $q=\left(q_{1}, q_{2}\right)$ of $Y$. The switched manifold $\Sigma=f^{-1}(0)$ is interpreted as the interrupt of each one of the forces 
(given by $y>0$ and $y<0$ ) to start the other. Thus

$$
Z(x, y)=\left\{\begin{array}{l}
\left(\begin{array}{cc}
-c_{1} & 0 \\
0 & \left(m c_{1}-\beta\right) / 2 m
\end{array}\right)\left(\begin{array}{l}
x-p_{1} \\
y-p_{2}
\end{array}\right)+\left(\begin{array}{c}
\left(y-p_{2}\right)^{2} \\
0
\end{array}\right) \text { if } y \geqslant 0, \\
\left(\begin{array}{cc}
-c_{2} & 0 \\
0 & \left(m c_{2}-\beta\right) / 2 m
\end{array}\right)\left(\begin{array}{l}
x-q_{1} \\
y-q_{2}
\end{array}\right)+\left(\begin{array}{c}
\left(y-q_{2}\right)^{2} \\
0
\end{array}\right) \text { if } y \leqslant 0 .
\end{array}\right.
$$

We have $Z \in B_{22}$ and $X(x, 0) \cdot Y f(x, 0)=\left(\frac{m c_{1}-\beta}{2 m}\right)\left(\frac{m c_{2}-\beta}{2 m}\right) p_{2} q_{2}$.

If $p_{2} q_{2}=0$ then by Lemma $5, Z$ is bounded. Now consider $p_{2} q_{2} \neq 0$. In this case no have tangent points $\left(\Sigma_{t}=\emptyset\right)$. If $p_{2} q_{2}>0$ then $\Sigma=\Sigma_{c}\left(\Sigma_{s}=\emptyset\right)$ and by item (b) of the Lemma $7, Z=(X, Y)$ is bounded. If $p_{2} q_{2}<0$ then $\Sigma=\Sigma_{s}\left(\Sigma_{c}=\emptyset\right)$ and by item (a) of the Lemma 7 we conclude that $Z=(X, Y)$ is bounded since $R_{22}^{p q}<0$ where

$$
R_{22}^{p q}=\frac{p_{2} \frac{m c_{1}-\beta}{2 m} c_{2}-q_{2} \frac{m c_{2}-\beta}{2 m} c_{1}}{-p_{2} \frac{m c_{1}-\beta}{2 m}+q_{2} \frac{m c_{2}-\beta}{2 m}} .
$$

\section{REFERENCES}

[1] B. Coll, A. Gasull And J. Llibre, Some Theorems on the Existence, Uniqueness, and Nonexistence of Limit Cycles for Quadratic Systems, J. Diff. Equations 67 (1987), 372-399.

[2] F. Dumortier And C. Herssens, Local Bifurcations and a Survey of Bounded Quadratic Systems, J. Diff. Equations 165 (2000), 430-467.

[3] R. Dickson And L. Perko, Bounded Quadratic Systems in the Plane, J. Diff. Equations 7 (1970), 251-273.

[4] A. F. Filippov, Differential Equations with Discontinuous Righthand Sides, Mathematics and its Applications (Soviet Series), Kluwer Academic Publishers-Dordrecht (1988).

[5] C. Li, J. Llibre, Z. Zhang, Weak Focus, Limit Cycles, and Bifurcations for Bounded Quadratic Systems, J. Diff. Equations 115 (1995), 193-223.

[6] L. Markus, Quadratic differential equations and non-associative algebras, Ann. Math. Studies, 45, Princeton Univ. Press (1960), 185-213.

(Jaume Llibre) Departament de Matemàtiques, Universitat Autònoma de Barcelona, Barcelona, Spain

(Clayton Eduardo Lente da Silva) Instituto de Biociências Letras e Ciências Exatas, Unesp - Univ Estadual Paulista, Deparatamento de Matemática, São José do Rio Preto, São Paulo, Brazil

(Paulo Ricardo da Silva) Instituto de Biociências Letras e Ciências Exatas, UNESP - Univ Estadual Paulista, Deparatamento de Matemática, São José do Rio Preto, SÃo Paulo, Brazil

E-mail address: jllibre@mat.uab.es

E-mail address: claedu@gmail.com

E-mail address: prs@ibilce.unesp.br 